Original research article

\title{
History-dependent patterns of whole ecosystems
}

\author{
Jonathan A. Sherratt* \\ Department of Mathematics and Maxwell Institute for Mathematical Sciences, Heriot-Watt University, Edinburgh EH14 4AS, UK
}

\section{A R T I C LE I N F O}

\section{Article history:}

Received 18 May 2012

Received in revised form 25 October 2012

Accepted 18 December 2012

Available online 22 February 2013

\section{Keywords:}

Pattern formation

Reaction diffusion advection

Hysteresis

Stability

Arid landscapes

Mussel beds

Wavetrain

\begin{abstract}
A B S T R A C T
Spatial patterns at the landscape scale have been documented in a wide variety of ecosystems across many parts of the world. Mathematical models have played an important role in understanding the causes of these patterns, and their implications for ecosystem change as environmental parameters vary. Preliminary results from simulation studies suggest the possibility of hysteresis, meaning that the wavelength and other properties of the pattern will vary in a history-dependent manner. This paper presents a detailed study of this phenomenon for two established models of landscape-scale patterns: the model of Klausmeier (Science 284 (1999) 1826-1828) for banded vegetation in semi-arid environments, and the model of van de Koppel et al. (American Naturalist 165 (2005) E66-E77) for patterning in young mussel beds. In both cases, the author demonstrates history-dependent patterns. Moreover, he shows how a knowledge of pattern existence and stability enables a detailed understanding of this hysteresis.
\end{abstract}

(c) 2013 Elsevier B.V. All rights reserved.

\section{Introduction}

Spatial patterns at the landscape scale were first reported via aerial photographs of sub-Saharan Africa, which revealed largescale patterns in which vegetation alternated with regions of bare ground (MacFadyen, 1950; Hemming, 1965). Spotted, striped and labyrinthine patterns of vegetation have now been documented in semi-arid parts of Africa, Australia, North and South America, and Asia (Valentin et al., 1999; Rietkerk et al., 2004; Deblauwe et al., 2008). Moreover, landscape-scale patterns are now known to occur in a wide variety of other ecosystems (see Rietkerk and van de Koppel (2008) for review), including patterns of open-water pools in peatlands (Belyea, 2007; Eppinga et al., 2009), banded and labyrinthine patterns in mussel beds (Paine and Levin, 1981; van de Koppel et al., 2005, 2008), regular isolated spots of trees and shrubs in savanna grasslands (Lejeune et al., 2002; Ben Wu and Archer, 2005), tussock patterns in freshwater marshes (van de Koppel and Crain, 2006; Yu, 2010), and striped patterns of tree lines ("ribbon forests") in the Rocky Mountains (Hiemstra et al., 2006; Bekker et al., 2009). Mathematical models have played an important role in understanding the ecological mechanisms responsible for these various patterns, and in predicting how they will change as environmental parameters vary. For vegetation patterns in semi-arid regions, many different mathematical

\footnotetext{
* Tel.: +441314513740.

E-mail address: jas@ma.hw.ac.uk
}

models have been proposed, reflecting different hypotheses on the key aspects of the underlying ecology. The papers of Lefever and Lejeune (1997), Klausmeier (1999), HilleRisLambers et al. (2001), von Hardenberg et al. (2010), Rietkerk et al. (2002) and Gilad et al. (2007) contain some of the key models, and a detailed review of the modelling literature in this area is given by Borgogno et al. (2009). For other landscape-scale patterns, mathematical modelling is less well established but has nevertheless made a significant contribution to our current understanding. A detailed review is outside the scope of this paper, but some examples help to illustrate this contribution. For peatland surface patterning, Eppinga et al. (2009) predicted that different mechanisms could be distinguished by data on the underlying patterns of nutrients and hydrology, but not by the surface patterns of vegetation and peat thickness. By comparing three alternative models for regular tussock spacing in a freshwater marsh, van de Koppel and Crain (2006) showed that scale-dependent inhibition provides the best explanation for the observed patterning. For savanna grasslands, Lejeune et al. (2002) used a mathematical model to show that the observation of isolated, regularly spaced vegetation patches can be explained via a Turing-like symmetry breaking instability. As a final example, van de Koppel et al. (2005) used a mathematical model to study the way in which spatial patterning in young mussel beds affects the overall mussel biomass, and the resilience of the mussel population to changes in the concentration of algae.

In a number of models for landscape-scale patterns, a given set of ecological parameters permits multiple pattern solutions, raising the possibility of hysteresis. Indeed, a preliminary 
simulation study by Sherratt and Lord (2007) demonstrated a history dependence of pattern wavelength in a model for banded vegetation in semi-arid environments. The present paper is a detailed study of this type of hysteresis. I consider two established models for landscape-scale patterning: the Klausmeier model for banded vegetation (Klausmeier, 1999), and the model of van de Koppel et al. (2005) for pattern formation in mussel beds. For both models, I demonstrate history-dependent patterning. Moreover, I will show that a detailed study of the existence and stability of pattern solutions provides an appropriate context for understanding the switches that occur in pattern wavelength as environmental parameters are varied.

In Section 2 I describe the phenomenon of banded vegetation patterning in semi-arid environments, and the model of Klausmeier (1999). I then discuss the use of the software package WAVETRAIN (http://www.ma.hw.ac.uk/wavetrain; Sherratt, 2012) to determine regions of parameter space in which there are pattern solutions, and additionally those regions in which these patterns are stable. In Section 3 a present an analogous discussion for mussel bed patterns, and the model of van de Koppel et al. (2005). In Sections 4 and 5 I describe simulation-based studies of the two models, demonstrating hysteresis and explaining the results using my wavetrain calculations. Finally in Section 6 I discuss the ecological implications of my results.

\section{Banded vegetation and the Klausmeier model}

Banded vegetation is by far the best documented type of landscape-scale pattern. It occurs on gentle slopes in semi-arid regions, with stripes of grass, shrubs or trees running parallel to the contours, alternating with regions of bare ground (Valentin et al., 1999; Rietkerk et al., 2004). Banded vegetation occurs in many parts of the world, with particularly detailed data from subSaharan Africa (MacFadyen, 1950; Valentin and d'Herbès, 1999; Couteron et al., 2000), Australia (Dunkerley and Brown, 2002; Berg and Dunkerley, 2004), and Mexico/South-Western USA (Montaña, 1992; McDonald et al., 2009). Typical wavelengths are about $1 \mathrm{~km}$ for trees and shrubs, with shorter wavelengths observed for grasses.

Many authors have used mathematical models to study banded vegetation, since there are no laboratory replicates, and field work is difficult and expensive; the modelling literature is reviewed by Borgogno et al. (2009). To be specific, I will consider only one model, due originally to Klausmeier (1999). A detailed ecological appraisal of this model is given by Ursino (2005), and mathematical properties of the equations are discussed by Sherratt (2005, $2010,2011,2013 a, b)$. The model consists of conservation equations for the plant biomass $u(x, t)$ and the water density $w(x, t)$, and when suitably non-dimensionalised it has the form

$$
\frac{\partial u}{\partial t}=\overbrace{w u^{2}}^{\begin{array}{c}
\text { plant } \\
\text { growth }
\end{array}}-\overbrace{B u}^{\begin{array}{c}
\text { plant } \\
\text { loss }
\end{array}}+\overbrace{\frac{\partial^{2} u}{\partial x^{2}}}^{\begin{array}{c}
\text { plant } \\
\text { dispersal }
\end{array}}
$$

$$
\frac{\partial w}{\partial t}=\underbrace{A}_{\begin{array}{c}
\text { rain } \\
\text { fall }
\end{array}}-\underbrace{w}_{\text {evaporation }}-\underbrace{w u^{2}}_{\begin{array}{c}
\text { uptake } \\
\text { by plants }
\end{array}}+\underbrace{v \partial w / \partial x}_{\begin{array}{c}
\text { flow } \\
\text { downhill }
\end{array}} .
$$

Here $t$ is time and $x$ is distance, measured in the uphill direction; the slope of the hillside is assumed to be constant. Although they represent a combination of ecological quantities, the (dimensionless) parameters $A, B$ and $v$ can be most usefully interpreted as reflecting rainfall, plant loss and slope gradient respectively. For full details of the dimensional model and the nondimensionalisation, see Klausmeier (1999) or Sherratt (2005).
For all parameter values, (1) has a stable "desert" steady state $u=0, w=A$. When $A \geq 2 B$, there are also two other homogeneous steady states:

$u=u_{1} \equiv \frac{2 B}{A-\sqrt{A^{2}-4 B^{2}}}, \quad w=w_{1} \equiv \frac{A-\sqrt{A^{2}-4 B^{2}}}{2}$

and $\quad u=u_{2} \equiv \frac{2 B}{A+\sqrt{A^{2}-4 B^{2}}}, \quad w=w_{2} \equiv \frac{A+\sqrt{A^{2}-4 B^{2}}}{2}$

The first of these (2) is always unstable to homogeneous perturbations, while (3) is linearly stable to homogeneous perturbations whenever $B<2$. For larger values of $B$ and small $A$, (3) can become unstable, giving complicated local dynamics including a limit cycle. However realistic parameter values for semi-arid environments imply that $B<2$ (Klausmeier, 1999), and I will restrict attention to this case. The linear stability analysis presented by Sherratt (2005) then shows that for some parameter values, (3) is unstable to spatially inhomogeneous perturbations, giving rise to patterns.

Fig. 1 shows an example such a pattern. The solution is periodic in space, with alternating peaks and troughs of $u$, corresponding to the vegetation bands and the gaps between them; there is a corresponding pattern of water density $w$. Fig. 1 also shows that the pattern moves at a constant speed in the uphill direction. Mathematically, this movement is a consequence of the advection term in (1b). In the ecological literature, there is a long-running debate on the migration of banded vegetation patterns (see Tonway and Ludwig (2001, pp. 24-26) for a detailed discussion). A considerable number of field studies do report uphill migration (Valentin et al., 1999, Table 5; Deblauwe, 2010, Chapter 10) with the proposed cause being higher moisture levels on the uphill edge

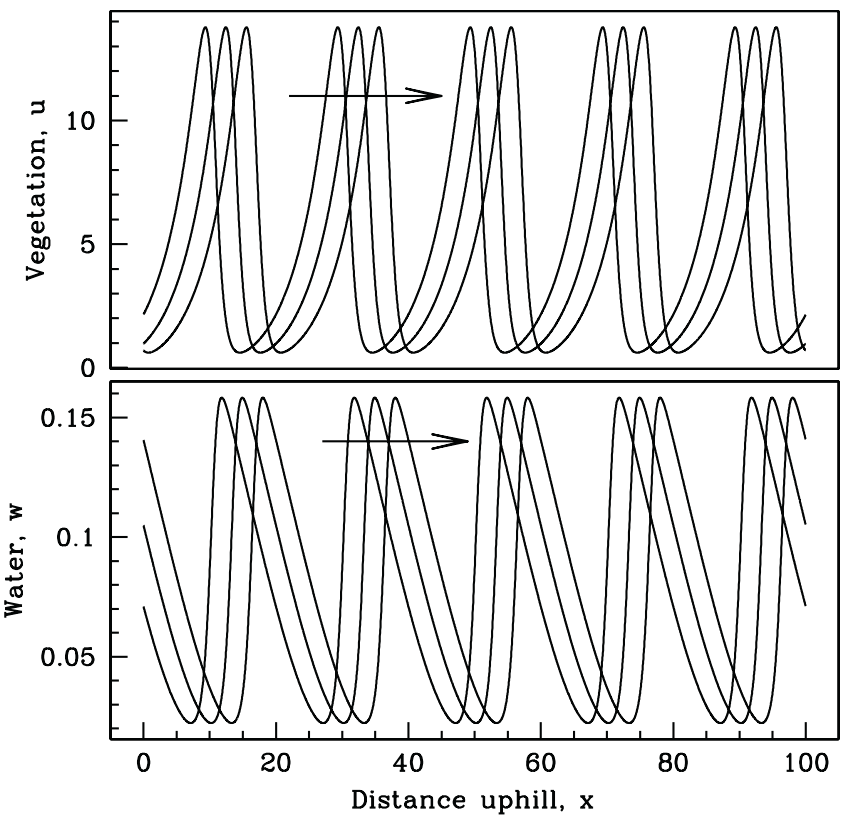

Fig. 1. A typical example of a pattern solution of the model (1) for vegetation in semi-arid environments. The alternating peaks and troughs of $u$ correspond to vegetation bands and the gaps between them, respectively. The solution is plotted at three different times, 3 dimensionless time units apart, to illustrate the uphill migration of the pattern. The parameter values are $A=2.3, B=0.45$ and $v=182.5$. The spatial domain is of length 100 with periodic boundary conditions. The initial conditions are small random perturbations (amplitude $\pm 5 \%$ ) to the vegetated steady state (3), and the first solution is plotted after 2400 dimensionless time units; this long time ensures that transients have decayed. The equations were solved numerically as described in Section 4 and Appendix B. 
of the bands compared to their downhill edge, leading to reduced plant death and greater seedling density (Montaña, 1992; Tongway and Ludwig, 2001). However, there is also contradictory data, reporting stationary patterns (Dunkerley and Brown, 2002; Mabbutt and Fanning, 1987; Couteron et al., 2000). Possible explanations for this include changes in the structure of bare soils that inhibit seed germination (Dunkerley and Brown, 2002), and preferential dispersal of seeds in the downhill direction, due to transport in run-off (Saco et al., 2007; Thompson and Katul, 2009).

The uphill migration of pattern solutions means that they are periodic travelling waves, and can be studied via the ansatz $u(x, t)=\tilde{u}(z)$ and $w(x, t)=\tilde{w}(z)$, where $z=x-c t$ and $c$ is the migration speed. Substituting these solution forms into (1) gives the travelling wave equations

$\frac{d^{2} \tilde{u}}{d z^{2}}+\frac{c d \tilde{u}}{d z}+\tilde{w} \tilde{u}^{2}-B \tilde{u}=0$

$\frac{(v+c) d \tilde{w}}{d z}+A-\tilde{w}-\tilde{w} \tilde{u}^{2}=0$.

Pattern solutions correspond to limit cycles in this system of oDES. General theory (Kopell and Howard, 1973; Sherratt and Smith, 2008) implies that if such pattern solutions exist for a given set of ecological parameters $A, B$ and $v$, then they will do so for a range of values of the migration speed $c$; the value of $c$ relevant to a particular case depends on the initial and boundary conditions. Therefore it is natural to consider the existence of pattern solutions in a parameter plane whose axes are the wave speed $c$ and one of the ecological parameters.

From an environmental viewpoint, the key control parameter is the rainfall, and an important role for mathematical models is to predict how ecosystems with banded vegetation will change in response to changes in rainfall. Therefore I focus attention on the $A-c$ parameter plane. The region of this plane giving patterns can be calculated using numerical bifurcation analysis. The new software package WAVETRAIN (http://www.ma.hw.ac.uk/wavetrain) is specifically designed for this purpose and can be used without any previous experience of numerical continuation. Full details of WAVETRAIN are given in Sherratt (2012), and I give only a brief summary of the overall approach. One begins by performing a loop over a grid of points in the $A-c$ parameter plane; for each parameter pair, WAVETRAIN determines whether or not there is a periodic travelling wave solution, i.e. a pattern. For any system of PDES, the parameter region giving patterns is bounded by either a locus of Hopf bifurcations in the travelling wave equations, a locus of homoclinic (infinite period) travelling wave solutions, or a locus of folds in the solution branch of periodic travelling waves. Using the results from the parameter grid run as a guide, WAVETRAIN can be used to locate and trace Hopf bifurcation and homoclinic loci, giving a detailed account of the parameter region giving patterns. Although this is a key step in understanding pattern solutions, it is of limited use by itself because it does not contain any information about stability. For any set of ecological parameters giving periodic travelling wave (pattern) solutions, these patterns will typically be stable for some values of $c$ and unstable for others. Here I use the term (un)stable to denote linear (in)stability on an infinite domain. Wavetrain can be used to determine the regions of stable and unstable patterns. Again, one first performs a loop over a grid of points in the parameter plane, calculating pattern stability in each case via numerical continuation of the essential spectra (Rademacher et al., 2007). Using these results as a guide, WAVETRAIN can then be used to trace the loci of boundaries in the parameter plane between stable and unstable patterns (Sherratt, in press).

Fig. 2 illustrates a typical result from these various calculations. The figure shows the region of the $A-c$ parameter plane in which

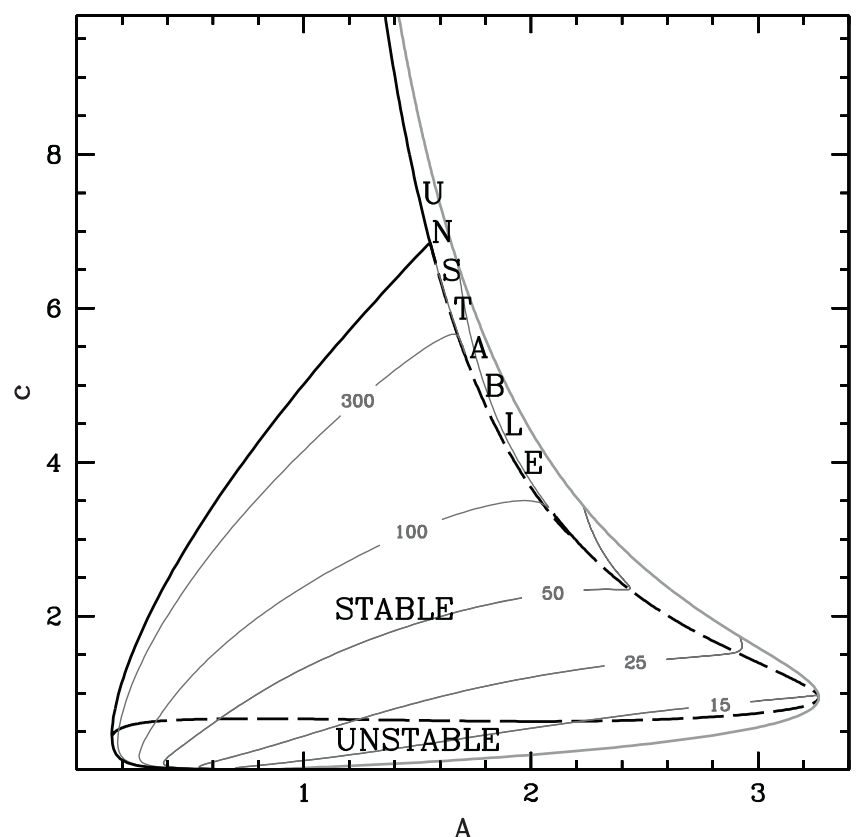

Fig. 2. Existence and stability of pattern solutions of the model (1) for vegetation in semi-arid environments, as a function of the rainfall parameter $A$ and the migration speed $c$, with $B=0.45$ and $\nu=182.5$. The thin lines are contours of constant pattern wavelength. The thick solid grey line is the locus of Hopf bifurcations of the nontrivial steady state (3) in the travelling wave equations (4). The thick solid black line is the an approximation to the locus of homoclinic solutions, given by the locus of solutions of a fixed large wavelength $(=2000)$. Patterns exist in the parameter region enclosed by these thick solid lines. However these patterns are only stable in the parameter region between the thick dashed black lines; outside this region the patterns are unstable. Details of the stability change are given in Appendix A. The plot has been truncated at $c=10$, and the parameter region giving patterns actually extends up to $c \approx 50$ (Sherratt, 2011). Moreover for $c$ above about 14 there is a fold in the solution branch as $A$ is varied with $c$ fixed, implying two different patterns for given values of $A$ and $c$ (see Sherratt (2011) for details); however, all patterns with $c>10$ are unstable as solutions of (1). There is also a fold in the solution branch as $A$ is varied with $c$ fixed at less than about 0.004 (Sherratt, 2013b); again this leads to two different pattern solutions, but both are unstable as solutions of (1). All calculations and plotting were done using the software package WAVETRAIN (http://www.ma.hw.ac.uk/wavetrain; Sherratt, 2012, in press; Rademacher et al., 2007). Full details of the WAVETRAIN input files, run commands and plot commands are given at http://www.ma.hw.ac.uk/ jas/supplements/hysteresis/.

patterns exist, and also where they are stable, for $B=0.45$ and $v=182.5$. The illustrated pattern region is bounded to the right by a locus of Hopf bifurcations of the steady state (3) in the travelling wave equations (4), and to the left by a locus of homoclinic solutions of (4). Note that the information on pattern existence (but not stability) in Fig. 2 has been presented previously by Sherratt and Lord (2007). Throughout the parameter region shown there is only one pattern solution. However for very large and very small values of $c$ there are small parameter regions with two different patterns for given values of $A$ and $c$ (see the figure legend for details).

\section{Mussel bed patterns and the van de Koppel model}

Mussel beds are a dominant feature of many intertidal regions. They are rarely uniform in space, and typically consist of complex irregular spatiotemporal patterns (Paine and Levin, 1981; Snover and Commito, 1998; van de Koppel et al., 2008). In these patterns, dense aggregations of mussels alternate with gaps that are usually colonised by subdominant species. The adaptive value of aggregation is that it reduces losses due to both predation and wave dislodgement; the latter benefit arises because nearby mussels are connected to one another via byssal threads (Denny, 1987). Although irregular patterns are the norm, regular banded patterns occur in beds of young blue mussels (Mytilus edulis) in the Wadden Sea, an 
intertidal region bordering the North Sea on the coastline of Denmark, Germany and The Netherlands. The bands are aligned perpendicular to the tidal flow, and have a wavelength of about $6 \mathrm{~m}$ (van de Koppel et al., 2005). The main food source for the mussel population in the Wadden Sea is algae. These reside primarily in the upper water layers, but there is some transport to lower layers where they become susceptible to predation by mussels. Algae are also advected with the tidal current. van de Koppel et al. (2005) proposed a model for mussel dynamics in the Wadden Sea, consisting of equations for mussel density $m(x, t)$ and algal concentration $a(x, t)$. When appropriately nondimensionalised (van de Koppel et al., 2005; Wang et al., 2009), the model has the form

$$
\frac{\partial a}{\partial t}=\overbrace{\alpha(1-a)}^{\begin{array}{c}
\text { transferto/ } \\
\text { from upper } \\
\text { water layers }
\end{array}}-\overbrace{a m}^{\begin{array}{c}
\text { consumption } \\
\text { by mussels }
\end{array}}+\overbrace{\frac{\beta \partial a}{\partial x}}^{\begin{array}{c}
\text { advection } \\
\text { by tide }
\end{array}}
$$

$$
\frac{\partial m}{\partial t}=\underbrace{\delta a m}_{\text {birth }}-\underbrace{\frac{\gamma m}{(1+m)}}_{\begin{array}{c}
\text { dislodgement } \\
\text { by waves }
\end{array}}+\underbrace{\frac{\partial^{2} m}{\partial x^{2}}}_{\begin{array}{c}
\text { random } \\
\text { movement }
\end{array}} .
$$

Here $t$ is time and $x$ is a spatial coordinate running away from the shore. The constant $\beta$ is positive, so that the algal advection term is unidirectional; this is because the incoming tide is the most important for the supply of algae to the mussel bed (van de Koppel et al., 2005). The term $\gamma /(1+m)$ reflects the reduction in the per capita loss rate due to the greater interconnectedness of mussels at higher densities, which gives greater resilience to dislodgement by waves. The most useful interpretation of the dimensionless parameters is that $\alpha$ reflects the exchange rate of algae between the upper and lower water layers, $\beta$ reflects the strength of the tidal flow, $\gamma$ reflects the maximum mussel loss rate, and $\delta$ reflects the algal concentration
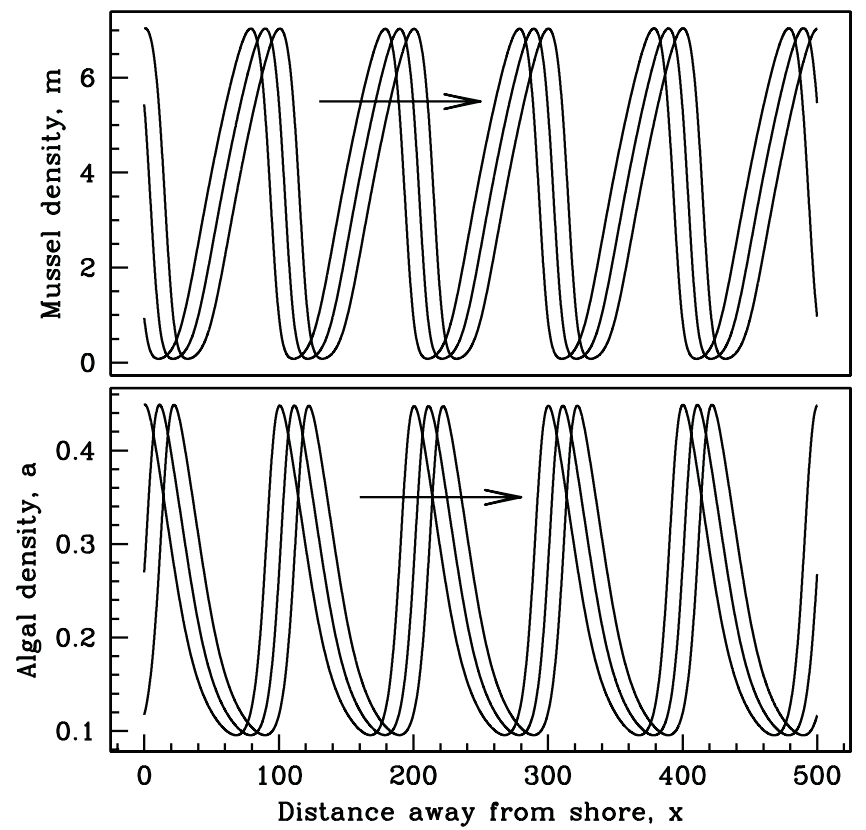

Fig. 3. A typical example of a pattern solution of the model (1) for mussel beds. The alternating peaks and troughs of $m$ correspond to bands of mussels and the gaps between them, respectively. The solution is plotted at three different times, 50 dimensionless time units apart, to illustrate the migration of the pattern away from the shore. The parameter values are $\alpha=0.6667, \beta=41.5692$ and $\gamma=0.1333$, $\delta=0.16$. The spatial domain is of length 500 with periodic boundary conditions. The initial conditions are small random perturbations (amplitude $\pm 5 \%$ ) to the steady state (6), and the first solution is plotted after $10^{4}$ dimensionless time units, which is sufficient for transients to have decayed. The equations were solved numerically as described in Section 5 and Appendix B. in the upper water layers. Eq. (5) were the first PDE model for mussel bed patterning; there are also a number of cellular automaton and individual-based models (e.g. Wootton, 2001; Guichard et al., 2003; van de Koppel et al., 2008; Robles et al., 2010). Recently Liu et al. (2012) have compared the predictions of (5) with those of a different PDE model, based on the observation that mussels feed more effectively on top of mussel-generated hummocks; this provides an alternative positive feedback mechanism.

For all parameter values, (5) has a steady state $a=1, m=0$, which is stable to both homogeneous and inhomogeneous perturbations when $\delta<\gamma$, and unstable otherwise. When $\delta>\gamma>\delta \alpha$ there is a second steady state

$a=\frac{\gamma-\delta \alpha}{\delta-\delta \alpha} \quad m=\frac{\alpha \delta-\alpha \gamma}{\gamma-\delta \alpha}$

The stability of this non-trivial steady state is parameterdependent, but for some parameter values it is stable to spatially homogeneous perturbations and unstable to inhomogeneous perturbations (see Wang et al., 2009, for details). Pattern formation then occurs, as illustrated in Fig. 3. As for the Klausmeier model discussed in Section 2, the patterns move at a constant speed; in this case the movement is away from the shore.

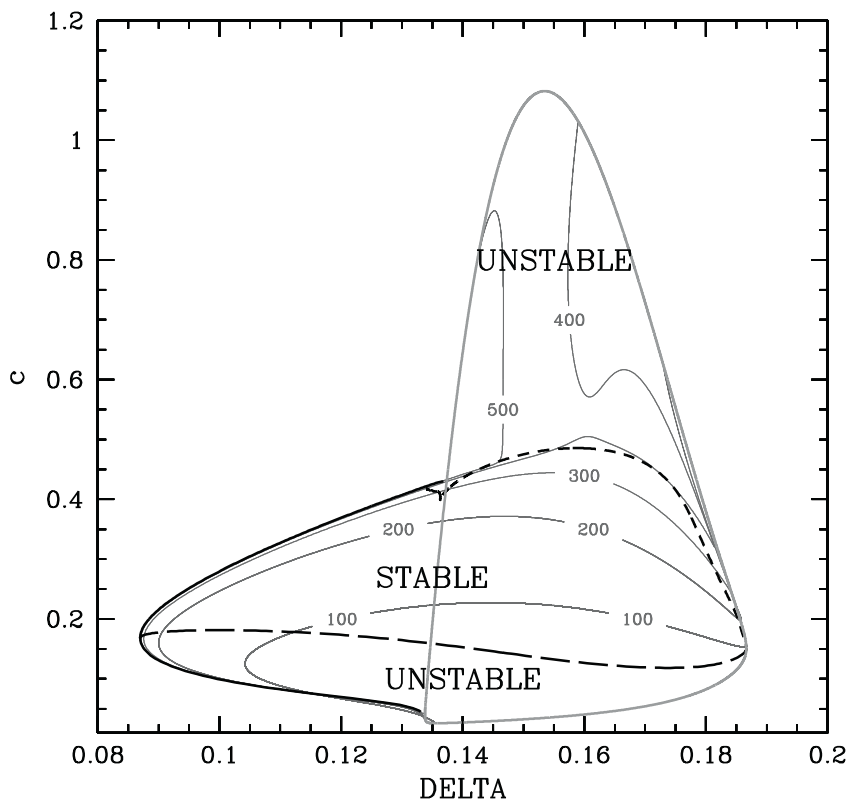

Fig. 4. Existence and stability of pattern solutions of the model (5) for mussel beds as a function of the algae supply $\delta$ and the migration speed $c$, with $\alpha=0.6667$, $\beta=41.5692$ and $\gamma=0.1333$. The thin lines are contours of constant pattern wavelength. The thick solid grey line is the locus of Hopf bifurcations of the nontrivial steady state in the travelling wave equations. The thick solid black line is an approximation to the locus of homoclinic solutions, given by the locus of solutions of a fixed large wavelength $(=1030)$. Patterns exist in the parameter region enclosed by these thick solid lines. However these patterns are only stable in the parameter region between the thick dashed black lines; outside this region the patterns are unstable. The short/long dashes indicate that the change in stability is of Hopf/ Eckhaus type (see Appendix A). The results in this figure concern patterns on the solution branch emanating from the right hand part of the Hopf bifurcation locus. For the migration speed $c$ between about 0.04 and 0.41 there is a second branch of pattern solutions emanating from the left hand part of the Hopf bifurcation locus. This solution branch is very localised in extent within the parameter plane (see Fig. 5a) and all of its patterns are unstable as solutions of (5). For $c$ above about 0.41 , the two solution branches connect. There is also a second region of parameter space containing multiple patterns, caused by a fold in the solution branch, roughly centred at $\delta \approx 0.125$ and $c \approx 0.06$ (illustrated in Fig. 5b). Both patterns are unstable as solutions of (5) in this region of the parameter plane. All calculations and plotting were done using the software package WAVETRAIN (http://www.ma.hw.ac.uk/ wavetrain; Sherratt, 2012, in press; Rademacher et al., 2007). Full details of the WAVETRAIN input files, run commands and plot commands are given at http:// www.ma.hw.ac.uk/ jas/supplements/hysteresis/. 
As in Section 2, the patterns can be studied via the travelling wave ordinary differential equations satisfied by the solution forms $a(x, t)=\tilde{a}(z), m(x, t)=\tilde{m}(z)$ where $z=x-c t$. Again, this can be done efficiently using the software package WAVETRAIN. The key ecological control parameter is $\delta$, which reflects the algal concentration in the upper water layers, and Fig. 4 illustrates the part of the $\delta-c$ parameter plane in which patterns exist, and also where they are stable, for $\alpha=0.6667, \beta=41.5692$ and $\gamma=0.1333$ (these parameter values are taken from Wang et al., 2009). All calculations and plotting were done using WAVETRAIN. The parameter region giving patterns is bounded to the right by a locus of Hopf bifurcations of the non-trivial steady state in the travelling wave equations, and to the left by a locus of homoclinic solutions for $\tilde{a}$ and $\tilde{m}$. Note that the information on pattern existence (but not stability) in Fig. 4 has been presented previously by Wang et al. (2009). As well as forming one boundary of the parameter region giving patterns, the Hopf bifurcation locus in Fig. 4 also runs through the middle of this region. An additional solution branch of patterns emanates from this part of the locus, which remains in the immediate vicinity of the Hopf bifurcation locus (Fig. 5a). It has a snaking form, so that there are a large (possibly infinite) number of coexisting patterns; however all are unstable as solutions of (5). There is also a small region of parameter space in which the pattern solution branch folds, implying multiple patterns (Fig. 5b), but again all of these solutions are unstable as solutions of (5).

From the viewpoint of this paper, the key significance of Figs. 2 and 4 is that they provide a template for understanding the results of numerical simulations of ( 1 ) and (5) as the control parameters $A$ and $\delta$ are varied.

\section{Hysteresis in the Klausmeier model}

The pattern shown in Fig. 1 was generated by applying a small spatially inhomogeneous perturbation to the vegetated steady state (3) of the Klausmeier model (1). In reality, the vegetation patterns that one sees in the field result from long series of transitions between different patterned states in response to environmental changes. With this in mind, I undertook a simulation study of the way in which the solution of (1) evolves as the rainfall parameter $A$ is varied. I used a domain of length 150 , and imposed periodic boundary conditions in order to eliminate boundary effects. An important precursor to my study is a detailed understanding of the accuracy of the numerical solution, and in Appendix B I present the results of convergence tests of my numerical method, which is a simple finite difference scheme. On the basis of these tests, I fixed the spatial grid spacing at 0.025 , with a time step of $1.096 \times 10^{-4}$; these imply a CFL number of 0.8 , and give an error of about $0.06 \%$ in the solution (see Table B. 1 ).

Fig. 6a illustrates the variation in the pattern speed and wavelength as $A$ is changed slowly. I used initial conditions of a small spatially inhomogeneous perturbation to the vegetated steady state (3), with $A=3.2$ which is just below the stability threshold. A pattern develops rapidly, with wavelength 15 . I then decreased $A$ in steps of 0.1 every 2400 time units until $A=0.5$, when I began a corresponding increase in $A$ back to 3.2. Note that there is no resetting of initial conditions when $A$ is changed: the results in Fig. 6 are for a single long simulation. The key phenomenon illustrated in Fig. 6a is hysteresis: for a given value of $A$, pattern selection depends on pattern history, with the patterns that occur when $A$ is decreased being different from those that occur for increasing $A$. The speed and wavelength shown in the figure are measured immediately before each change in $A$. As $A$ is decreased below 3.2 the speed of the pattern changes, and there is a corresponding change in pattern form; in particular the mean value of the plant density $u$ decreases, as one would expect intuitively as a consequence of reduced levels of rainfall (Fig. 6b). However the wavelength initially remains constant as $A$ is decreased, until there is an abrupt shift in wavelength from 15 to $150 / 7 \approx 21.428$ at $A=2.0$ (note that the periodic boundary conditions imply that any pattern must have a wavelength for
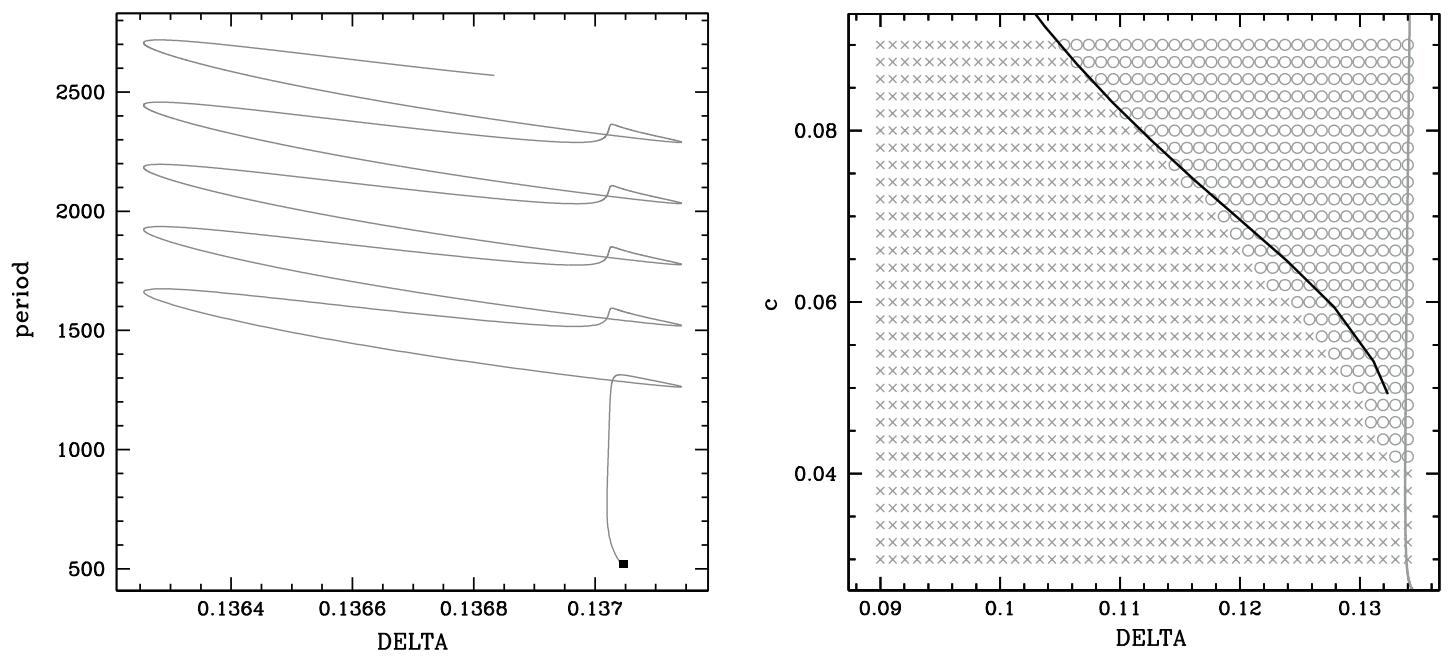

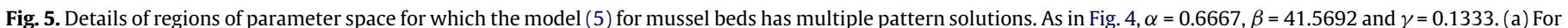

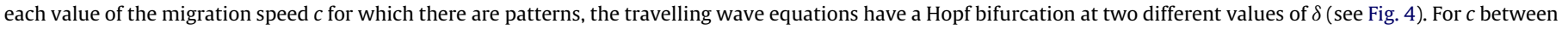

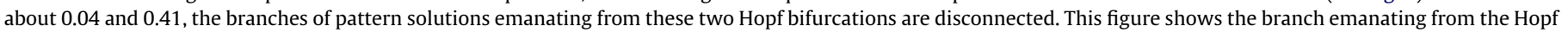

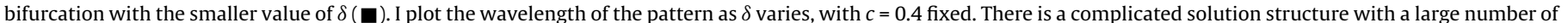

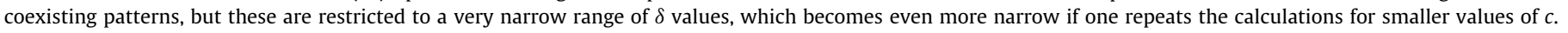

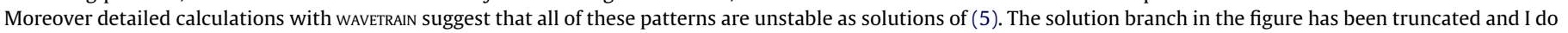

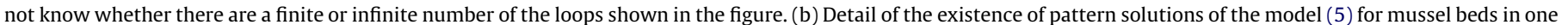

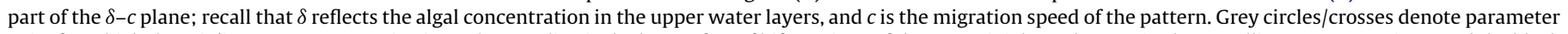

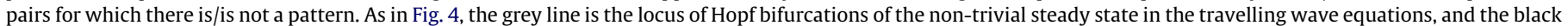

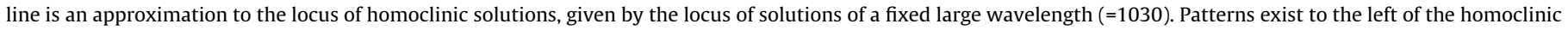

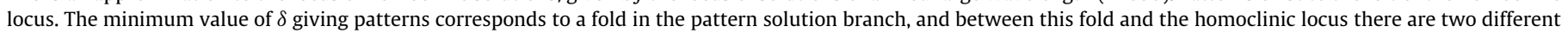

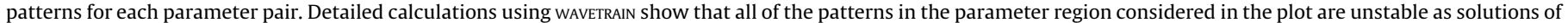

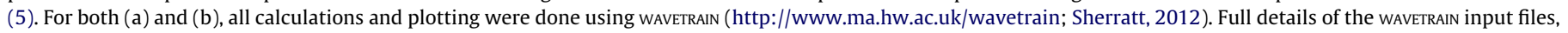
run commands and plot commands are given at http://www.ma.hw.ac.uk/ jas/supplements/hysteresis/. 


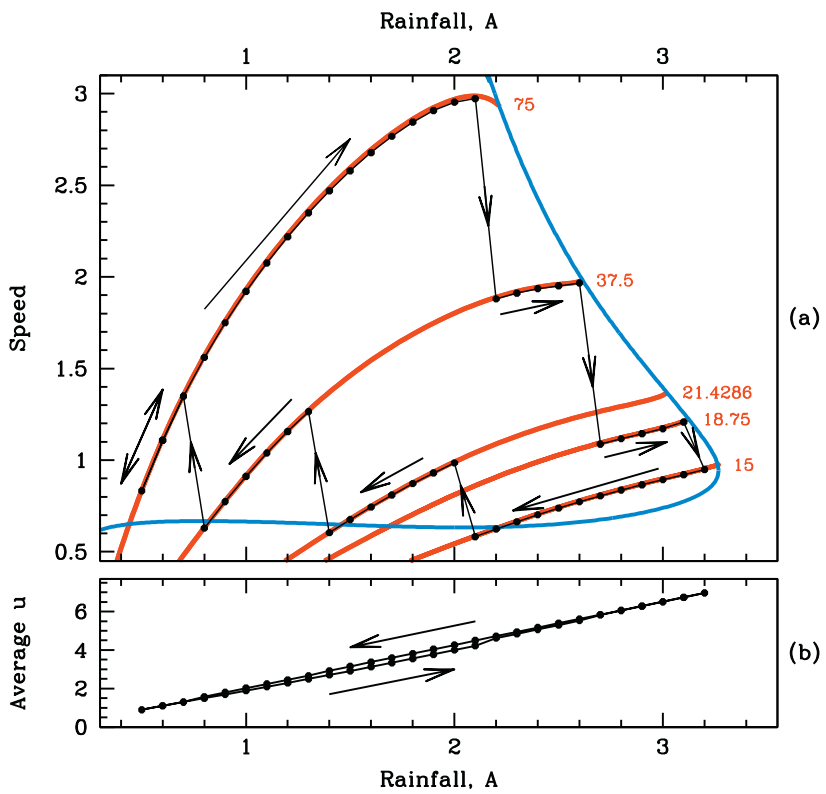

Fig. 6. Changes in patterns in the model (1) for vegetation in semi-arid environments, as the rainfall parameter $A$ is varied slowly, with $B=0.45$ and $v=182.5$, on a domain of length 150 . I decrease $A$ from 3.2 to 0.5 in steps of 0.1 , and then increase it again, running for 2400 time units at each value of $A$. Note that there is no resetting of initial conditions when $A$ is changed: the results in the figure are for a single long simulation. The boundary conditions are periodic, and the initia condition is low amplitude noise applied to the vegetated steady state. For the resulting patterns, the black dots and thin black lines show (a) the wavelength and speed, and (b) the average value of $u$, immediately before each change in $A$; the arrows indicate the direction in which $A$ is changing. The results provide a clear demonstration of hysteresis in pattern selection, confirming the results of Sherratt and Lord (2007). The dashed dark grey line (solid blue online) shows the boundary of the parameter region in which pattern solutions are stable. The thick light grey lines (red online) show contours of constant pattern wavelength. The numbers beside the curves show the corresponding wavelengths, all of which have 150 as an integer multiple; this condition on the wavelengths is a consequence of the periodic boundary conditions. Any wavelength $150 / n\left(n \in \mathbb{Z}^{+}\right)$could arise in the solution, but in fact only $n=2,4,7,8,10$ occur in this simulation. Wavelength contours for other values of $n$ are omitted from the plot to aid visual clarity. Also in the interests of clarity, I only plot the sections of these contours lying below the upper part of the stability boundary. The wavelength contours and stability boundary were calculated using the software package WAVETRAIN, as in Fig. 2. The solution follows a contour of constant wavelength until this leaves the parameter region giving stable waves, when there is a transition to a new wavelength. Note that at some stages of the simulation, a pattern arises that lies just outside the stability region; this is discussed in the main text. Details of the numerical method are given in Appendix B. Users considering reproducing this figure should note that the simulation took about 15 days on a Linux PC with a $2.83 \mathrm{GHz}$ Intel Core 2 Quad Q9500 processor.

which 150 is an integer multiple). The wavelength then remains constant at 21.428 while the speed and the mean value of $u$ decrease, until a second change in wavelength occurs, to 150/ $4=37.5$, at $A=1.3$. Corresponding behaviour then repeats as $A$ is decreased further, and also when $A$ is increased.

In Fig. 6a, I superimpose on the simulation data both the relevant contours of constant pattern period, and also the boundary of the region of pattern stability; these were shown previously in Fig. 2 and their calculation was discussed in Section 2. The abrupt changes that occur in pattern wavelength correspond to the period contours crossing the stability boundary. Thus the pattern remains at a constant wavelength until the pattern with that wavelength loses stability. A new wavelength is then selected, and I have been unable to identify any systematic basis for this selection.

A notable feature of Fig. 6a is that some of the observed patterns lie just outside the stable region of parameter space. For example, as $A$ is decreased through 2.1 the pattern remains at wavelength 15 (with $c \approx 0.58$ ) even though this solution lies in the unstable region. There are two separate potential causes of this apparent discrepancy. Firstly, the division into stable and unstable patterns in the figure refers to an infinite domain, whereas the simulations are done on a finite domain (length 150). This puts a restriction on the possible linear modes that are admissible when investigating stability, and therefore increases the region of parameter space giving stability. To illustrate this, I performed a new numerical study, in which I decreased $A$ from 2.4 in steps of 0.05 , with a time interval of 2500 between decrements. The initial solution was the pattern for $A=2.4$ with wavelength 15 , which I calculated using WAVETRAIN. I performed this simulation on domains of length $15 \mathrm{~N}$ for a variety of integer values of $N$, recording in each case the first value of $A$ at which the solution is no longer a pattern of wavelength 15 . The results are illustrated in Fig. 7a. For $N=1$ there is no result indicated in the figure because the pattern remains of wavelength 15 as $A$ is decreased down to 1.0 , at which point I ended the simulation. As $N$ is increased above 1 , there is an increase in the value of $A$ at which the wavelength changes, reflecting the shrinkage of the parameter region giving stable patterns.

Simulations on very small domains are of limited relevance to real instances of banded vegetation. In order to be observable in the field the patterns must span a number of wavelengths, and in practice patterned areas are typically quite extensive. Therefore the enlargement of the parameter region giving stable patterns due to the finiteness of the domain is a minor effect in practice. Moreover, numerical evidence indicates that for $A=2.1$ the pattern with wavelength 15 is in fact unstable on a domain of length 150 with periodic boundary conditions. I draw this conclusion from simulations with initial conditions consisting of low amplitude noise applied to a pattern calculated using WAVETRAIN.

The explanation for a pattern of wavelength 15 being observed for $A=2.1$ in Fig. 6 must therefore lie elsewhere, and the cause is in fact the length of time between changes in the value of $A$. When a pattern lies just outside the stable parameter region, the unstable linear modes will grow very slowly. If the time scale of this growth is significantly longer that the interval between changes in $A$, then the instability will not be manifested in the simulation results. This is illustrated in Fig. 7b. For this figure, I solved (1) on a domain of length 300 , again decreasing $A$ from 2.4 in steps of 0.05 with the initial solution being the pattern for $A=2.4$ with wavelength 15 , which I calculated using WAVETRAIN. For various values of the time interval between decrements in $A$, I again recorded the first value of $A$ at which the solution is no longer a pattern of wavelength 15 . The results show that the time interval has a significant effect on the variation in pattern form.

The nondimensionalisation and parameter estimates of Klausmeier (1999) imply that one dimensionless time unit corresponds to about 0.25 years. Therefore the (dimensionless) time interval of 2400 between changes in $A$ that is used in Fig. 6 corresponds to about 600 years. Fig. 7b shows that 2400 is already sufficiently short to have an impact on the simulation results; nevertheless, it is too long to be ecologically relevant. A gap of 600 years between changes in $A$ means that the entire simulation shown in Fig. 6 takes about 30000 years: over such a long period there will be major shifts in global climate that make a simple model such as (1) inappropriate. Nevertheless I deliberately used such a long time interval for the figure because it facilitates understanding of the relationship between the simulation results and the calculations of pattern existence and stability.

Figs. 8 and 9 show the results of the corresponding simulations with the time interval between changes in $A$ reduced to 240 and 40, which correspond to about 60 and 10 years respectively. In both cases the overall form of the solution is again gradual shifts in pattern form with the wavelength remaining constant, punctuated 

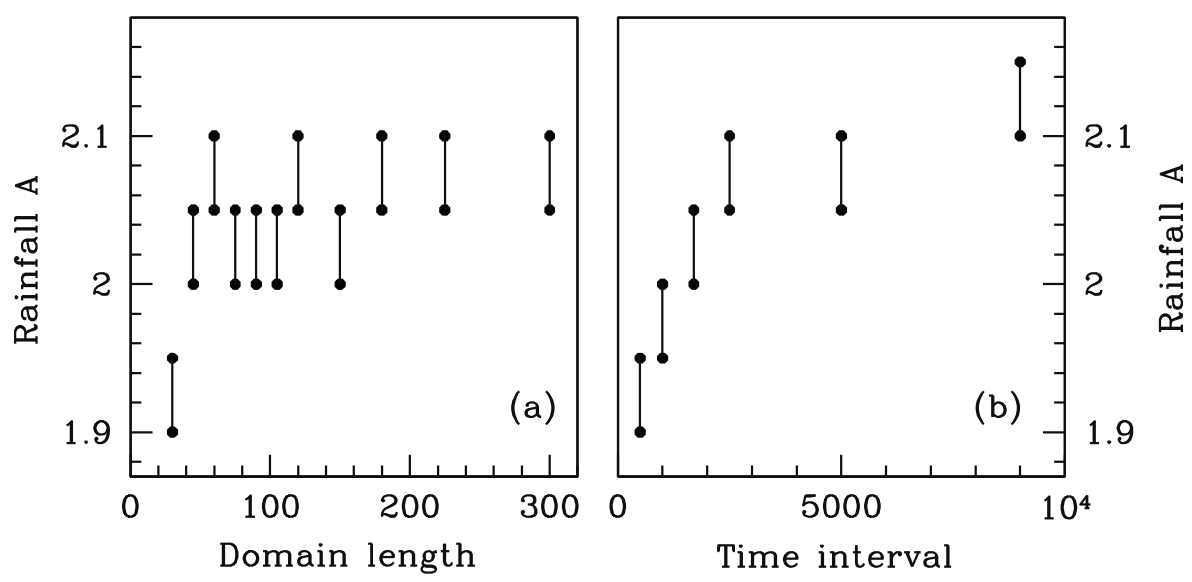

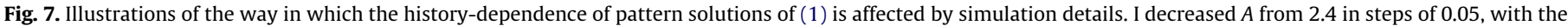

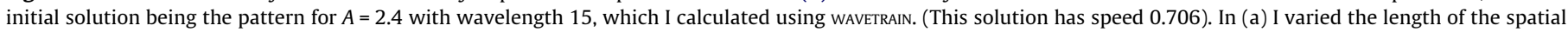

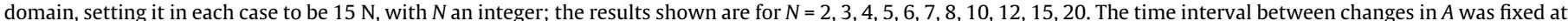

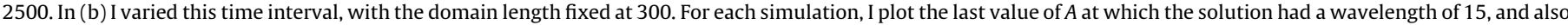

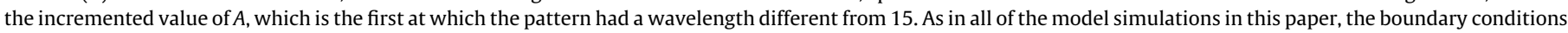

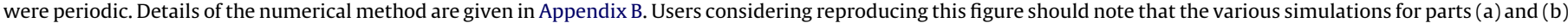
took totals of 21 days and 32 days respectively, on a Linux PC with a $2.83 \mathrm{GHz}$ Intel Core 2 Quad Q9500 processor.

by abrupt shifts in wavelength. Also the average value of plant density $u$ is again a monotonic function of the rainfall $A$, as one would expect intuitively. However the relatively short time intervals between changes in $A$ means that some of the abrupt shifts in pattern wavelength lie significantly outside the parameter region giving stable waves. Note that in Fig. 8, the wave speed recorded as $A$ is decreased through 0.9 does not lie on any of the contours of constant period. The solution at this point resembles a pattern of wavelength 50 but has not fully converged to the pattern solution by the end of the time interval of 240 . A similar phenomenon occurs in Fig. 9 as $A$ is increased through 2.1, and even more strikingly in the first two simulation outputs, at $A=3.2$ and 3.1. In fact in this case the solution does not have the appearance of a fully periodic pattern until $A=2.8$.

The results of this section can be summarised as follows. Gradual changes in the rainfall parameter $A$ result in a
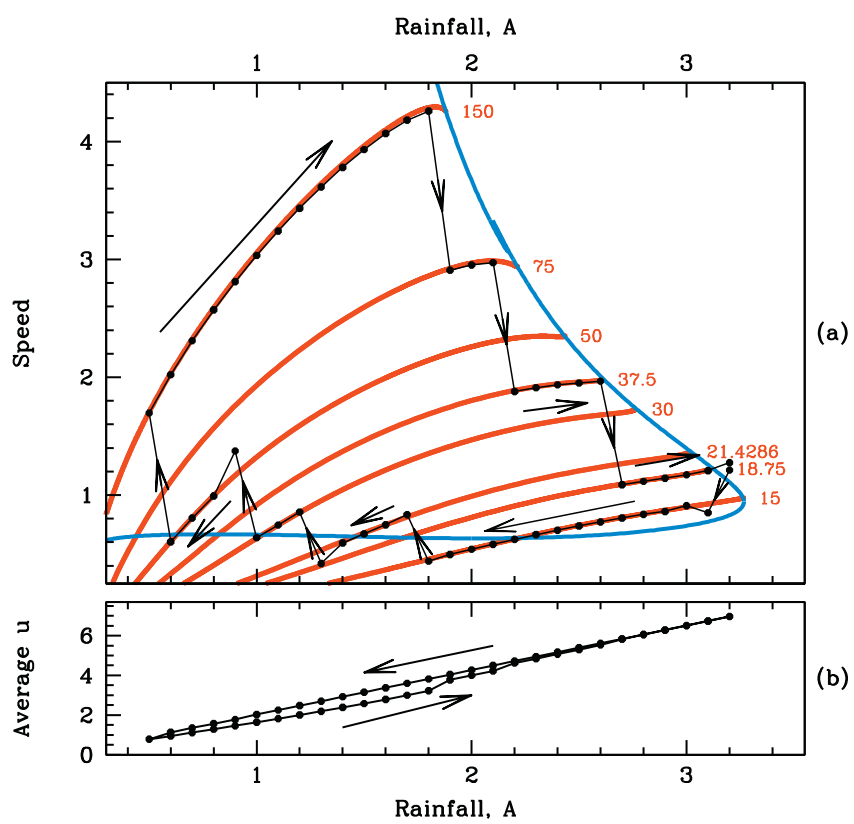

Fig. 8. The results of a simulation identical to that used for Fig. 6, except for the time interval between changes in $A$ being 240 . characteristic form of pattern variation, with wavelength remaining constant except for occasional abrupt switches. When the domain length and the time interval between changes in $A$ are both large, the switches in wavelength occur when the contour of constant wavelength crosses the boundary of the parameter region for stable patterns. Reductions in either the domain length or the time interval cause the switches to move outside the stability boundary. In practice, parameter estimates for banded vegetation in semi-arid environments mean that the domain length is sufficiently large that its effect on switch location is not significant, but appropriate values of the time interval between changes in rainfall level are relatively short, and cause the switches in wavelength to lie some distance outside the parameter region for stable patterns.

The slow timescale over which pattern form responds to changes in rainfall level has an important implication for the use of

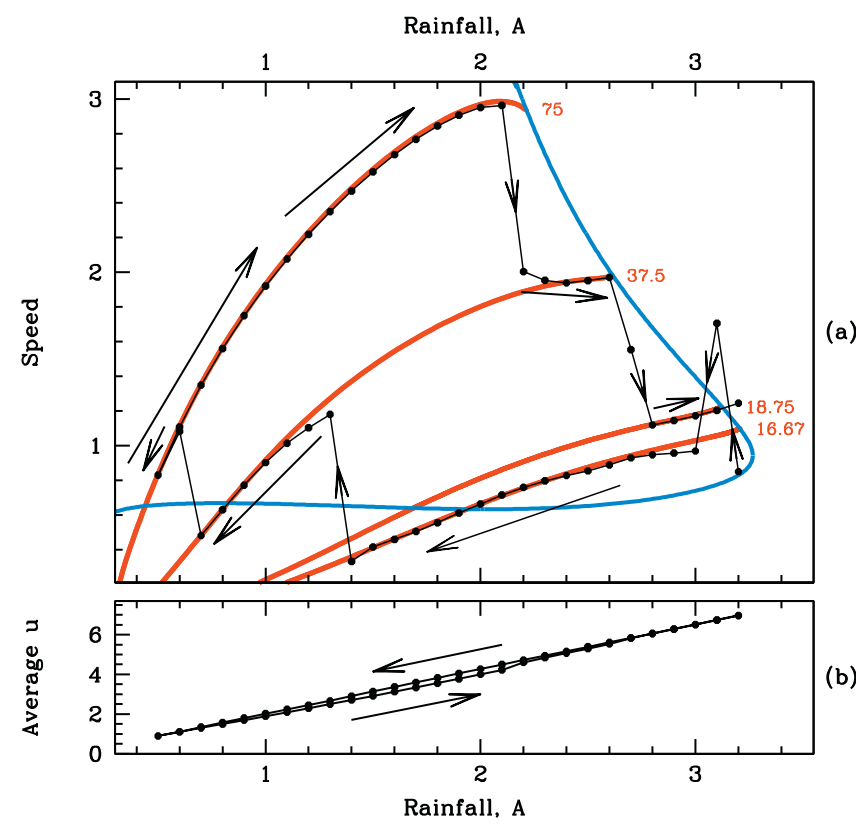

Fig. 9. The results of a simulation identical to those used for Figs. 6 and 8, except for the time interval between changes in $A$ being 40 . 
patterns as predictors of catastrophic shifts in ecosystems. In a highly influential paper, Rietkerk et al. (2004) reviewed evidence that self-organised patchiness is frequently a prelude to irreversible shifts between ecosystem states. For example, as rainfall decreases in semi-arid environments, there is a transition from homogeenous to patterned vegetation, and then at lower rainfall levels there is a second and irreversible transition to unvegetated desert. Rietkerk et al. then argued that ecosystem patterning had an important potential role as an indicator of subsequent catastrophic shifts. This argument was a theoretical one, not supported by simulations of any particular model, and it assumes that the current vegetation pattern reflects the current environmental state. My results suggest that for semi-arid environments this assumption may be invalid for reasonable rates of environmental change, and that the predictive power of pattern states is in fact significantly undermined by the slow timescale at which patterns respond to changes in rainfall level.

\section{Hysteresis in the van de Koppel model}

For the van de Koppel model (5) for mussel bed patterning, Fig. 4 shows that crossings by wavelength contours of the pattern stability boundary are concentrated at smaller values of $\delta$, and therefore I focused my investigation of pattern hysteresis on this parameter region. Fig. 10 shows the results of varying $\delta$ from 0.15
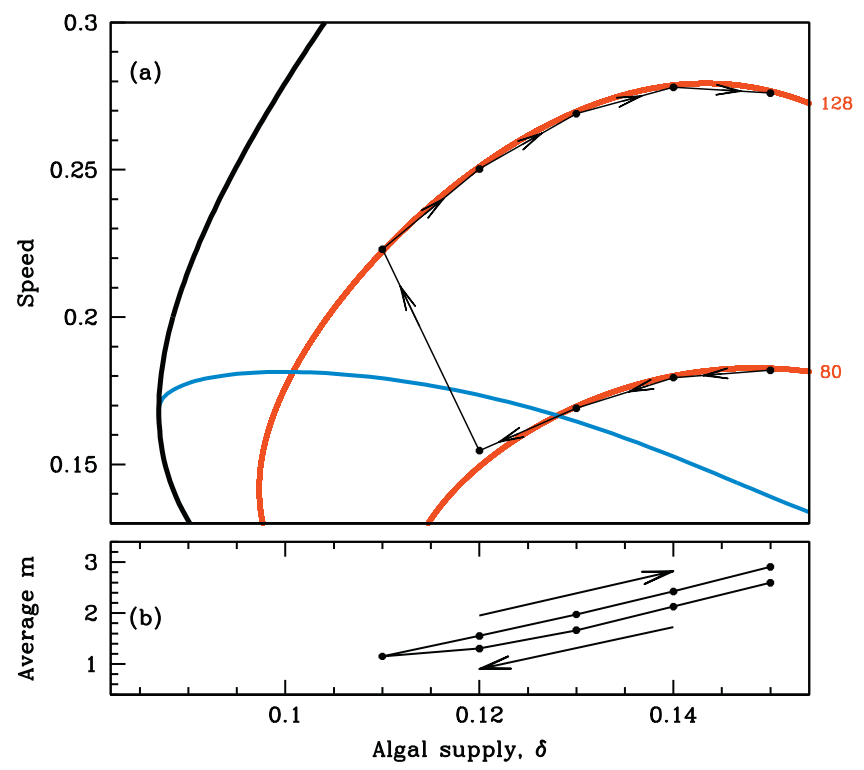

Fig. 10. Changes in patterns in the model (5) for mussel beds as the algal supply parameter $\delta$ is varied slowly, with $\alpha=0.6667, \beta=41.5692$ and $\gamma=0.1333$, on a domain of length 1920 . I decrease $\delta$ from 0.15 to 0.11 in steps of 0.01 , and then increase it again, running for $5 \times 10^{4}$ time units at each value of $\delta$. There is no resetting of initial conditions when $\delta$ is changed, and the results in the figure are for a single long simulation. The boundary conditions are periodic, and the initial condition is the pattern solution of wavelength 80 for $\delta=0.15$, which I calculated using WAVETRAIN (Sherratt, 2012). For the resulting patterns, the black dots and thin black lines show (a) the wavelength and speed, and (b) the average value of the mussel density $m$, immediately before each change in $\delta$; the arrows indicate the direction in which $\delta$ is changing. The results provide a clear demonstration of hysteresis in pattern selection. The dashed dark grey line (solid blue online) shows the boundary of the parameter region in which pattern solutions are stable. The thick light grey lines (red online) are contours of pattern wavelength 80 and 128 . The wavelength contours and stability boundary were calculated using the software package WAVETRAIN, as in Fig. 4. The solution follows the wavelength 80 contour until this leaves the parameter region giving stable waves, when there is a transition to the new wavelength of 128 . Note that as $\delta$ is decreased through 0.12 , the solution has not yet settled to a new pattern solution (see Fig. $6 \mathrm{c}$ ). Details of the numerical method are given in Appendix B. Users considering reproducing this figure should note that the simulation took about 15 days on a Linux PC with a $2.83 \mathrm{GHz}$ Intel Core 2 Quad Q9500 processor. to 0.11 in steps of 0.01 , and then increasing it again. Recall that the parameter $\delta$ reflects the algal concentration in the upper water layers. As an initial condition for $\delta=0.15$, I used the pattern solution of wavelength 80 , which I calculated using WAVETRAIN. Note that there is no resetting of initial conditions when $\delta$ is changed: the results in Fig. 10 are for a single long simulation. In view of the dependence of my results for the Klausmeier model on the domain length and the time interval between parameter changes, I took both of these to be relatively large: the domain length was $1920=80 \times 24$, and the time interval was $5 \times 10^{4}$. In contrast to the Klausmeier model, a long dimensionless time interval such as this is ecologically relevant in this case: the nondimensionalisation and parameter estimates of van de Koppel et al. (2005) imply that a dimensionless time interval of $5 \times 10^{4}$ corresponds to about 14 days.

The behaviour illustrated in Fig. 10 is directly analogous to that described in Section 4 for the Klausmeier model. As $\delta$ is decreased the pattern speed changes in order to keep the wavelength constant at 80 , until the wavelength contour crosses the stability boundary. There is then a transition in pattern wavelength to 128 . When $\delta$ is then increased, the wavelength remains at 128: again this is an instance of hysteresis.

As well as demonstrating these similarities between the Klausmeier model (1) for semi-arid landscapes and the van de Koppel model (5) for mussel beds, my various simulations have also revealed an important difference. In simulations with shorter time intervals between changes in $\delta$, it is common for the simulated mussel population to die out when the contour of the current pattern wavelength crosses the stability boundary, rather than a new pattern wavelength developing. This is illustrated in Fig. 11, for which all simulation details are the same as for Fig. 10 except that the time interval between changes in $\delta$ was halved to $2.5 \times 10^{4}$, which corresponds to about 7 days. As in Fig. 10, the solution proceeds along the wavelength 80 contour until this crosses the stability boundary; in fact a pattern of wavelength 80 also occurs just beyond the stability boundary, due to the finiteness of the domain and of the time interval between changes in $\delta$ (see Section 4). However, the mussel population density then simply decays to zero, and it remains at (almost) zero as $\delta$ is increased again. Again this is an example of hysteresis in the mussel density,
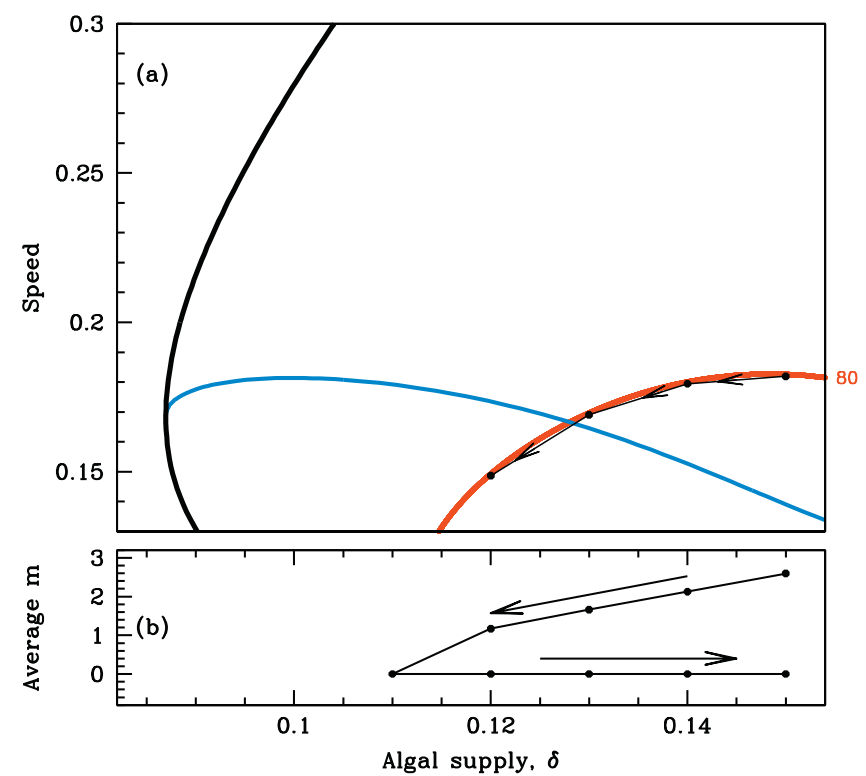

Fig. 11. The results of a simulation identical to that used for Fig. 10 except for the time interval between changes in $\delta$ being $2.5 \times 10^{4}$. The mussel population dies out when $\delta=0.11$ and does not recover when $\delta$ is increased again. 

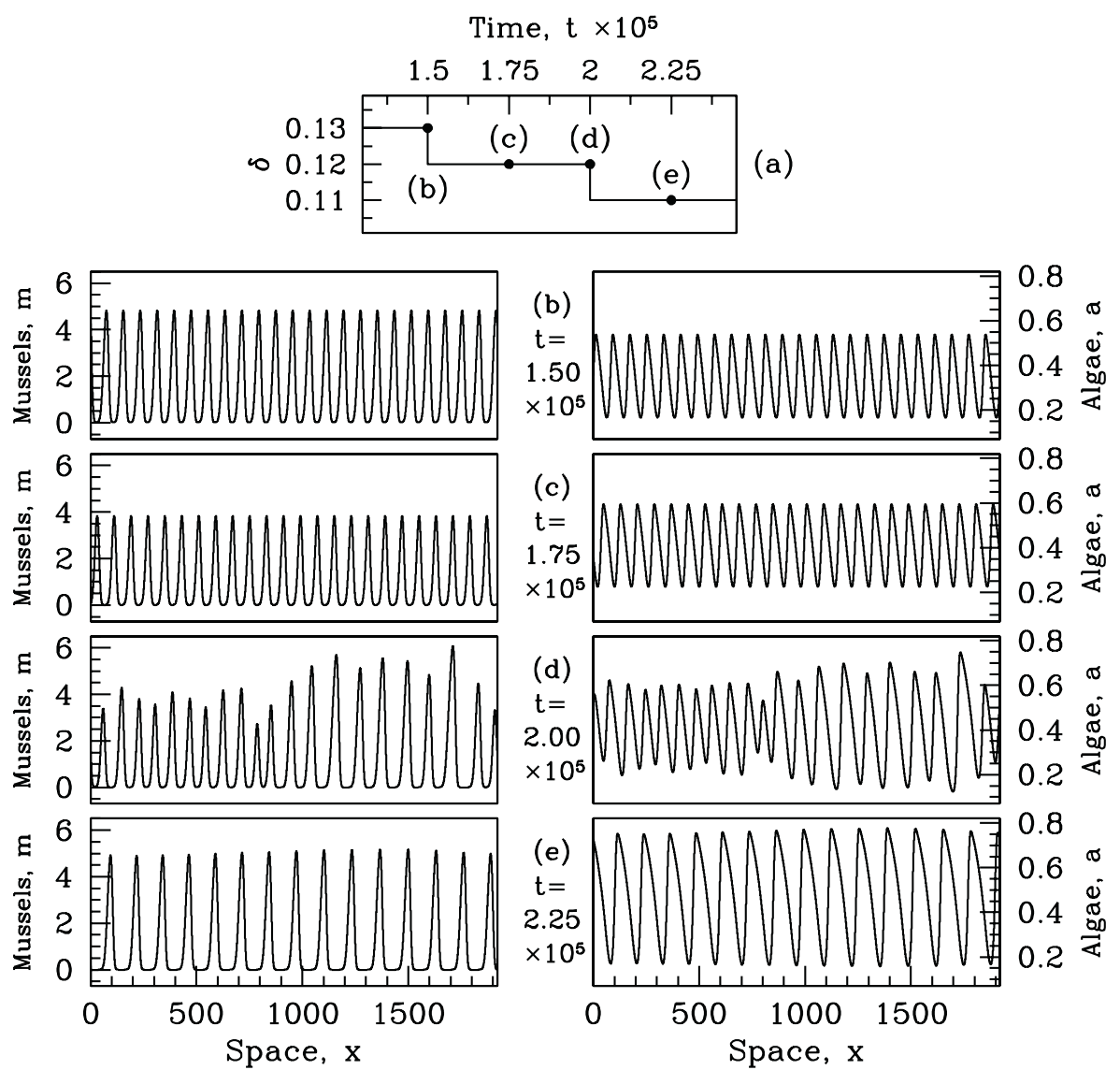

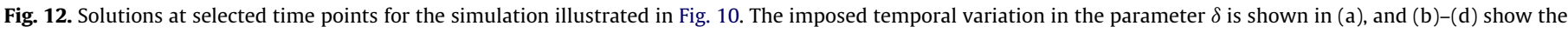

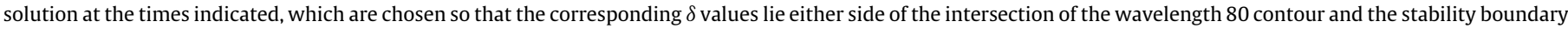

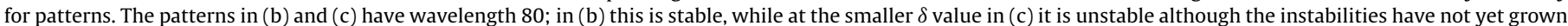

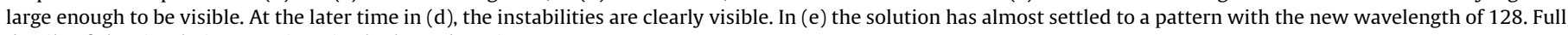
details of the simulation are given in the legend to Fig. 10.

but involving a pattern and the mussel-free state, rather than patterns of different wavelengths. During my extensive series of simulations for the Klausmeier model (1), I have only observed the plant-free state when the rainfall parameter $A$ is reduced sufficiently far that no patterns are possible.

The trivial ("mussel-free") steady state of the van de Koppel model is locally stable to both homogeneous and inhomogeneous perturbations when $\delta<\gamma$. Therefore once stability of a pattern with a particular wavelength is lost with $\delta<\gamma$, the trivial state and a pattern of a different wavelength are both possible new solutions, in principle. The same is true for the Klausmeier model, for which the trivial ("desert") steady state is locally stable to both homogeneous and inhomogeneous perturbations for all parameter values. The selection between these outcomes will depend on the size of the basin of attraction of the trivial state, relative to those of the stable patterns. The implication of my results is that the mussel-free state has a relatively large basin of attraction in the van de Koppel model (at least for $\delta=0.11$ ), while that of the desert state in the Klausmeier model is relatively small. I have not attempted to quantify these basins of attraction, and this would be a natural target for future work, with the main difficulty being the computational expense of the large number of simulations that would be required. Another natural area for future work would be to compare the results I have described for the van de Koppel model (5) with corresponding predictions from the model of Liu et al. (2012), which is based on the increased efficiency of mussel feeding on top of mussel-generated hummocks. This would help to highlight key similarities and differences between patterns generated by the different positive feedback mechanisms that are represented in the two models.

It is instructive to compare the behaviours in Figs. 10 and 11 after the wavelength 80 contour crosses the pattern stability boundary, and this is done in Fig. 12 . At $\delta=0.12$ the pattern of wavelength 80 is unstable, even on the finite domain of length 1920 which is used in both figures. However, the growth rate of the unstable modes is very small. Over a dimensionless time period of 25000 , the solution does not deviate significantly from a pattern of wavelength 80 (Fig. $12 \mathrm{~b}$ and c). In Fig. $11, \delta$ is then changed to 0.11 , for which the pattern of wavelength 80 lies within the basin of attraction of the mussel-free state, so that the mussel population collapses. However in Fig. $10, \delta$ remains at 0.12 for a further dimensionless time of 25,000 . This is sufficiently long to allow the unstable modes to grow and dominate the solution (Fig. 12d). For $\delta=0.11$, this irregular transient solution lies in the basin of attraction of a pattern of wavelength 128 (Fig. 12e), rather than the mussel-free state.

\section{Discussion}

I have demonstrated history-dependent landscape-scale patterning in two established mathematical models. A natural question is whether such history dependence occurs in real ecosystems, and the answer is that I am not aware of any data indicating this phenomenon. However there are very few data sets on landscape-scale patterns that cover an appropriate span of time. For vegetation in semi-arid environments, a number of authors 
have presented data on pattern dynamics. Some of these studies involve measurements of pattern location relative to ground benchmarks (Worrall, 1959; Leprun, 1999), while others use aerial photographs (Barbier et al., 2006; Lesschen et al., 2008) or satellite images (Deblauwe, 2010; Deblauwe et al., 2011). However for banded patterns, almost all studies simply compare the patterns at two time points, in order to assess the extent of uphill migration. A notable exception is the work of Valentin and d'Herbès (1999), who compared data from several sites in south-western Niger, collected on 6 occasions between 1950 and 1995. The most recent data set was from ground measurements, with the previous 5 being from aerial photographs. They looked specifically at the way in which the pattern varies with rainfall, and showed that the wavelength did indeed remain constant, with the widths of the vegetation bands and the bare interbands changing so that their ratio is an increasing function of rainfall. These results are in direct accord with my simulations, but since the pattern wavelength remains constant during the study period, this data set does not permit an assessment of history-dependence. For mussel beds, the only data that I am aware of on banded patterns comes from aerial photographs taken above the Wadden Sea on two different days, one in 1994 and the other in 2001 (van de Koppel et al., 2005). The patterns in these two data sets are completely unrelated: mussel beds in the Wadden Sea are either removed or severely damaged by Winter storms, so that considerations of pattern dynamics are only meaningful within a single year. In contrast to vegetation patterns in semi-arid regions, it has been possible to replicate selforganised patterns of mussel beds in laboratory settings (van de Koppel et al., 2008); however the patterns are labyrinthine rather than banded.

In view of this lack of relevant field data, what is the ecological significance of the work in this paper? The answer is that it provides a focal point for future data gathering and analysis. As a consequence of climate change, environmental parameters are liable to much greater variation now and in the near future than has previously been the case. My results suggests that one consequence of this will be history-dependent patterning. Moreover the sophisticated methods of data acquisition and analysis that are now available at the whole ecosystem scale offer real prospects of identifying occurrences of pattern hysteresis in the field.

\section{Acknowledgements}

This work was supported in part by a Leverhulme Trust Research Fellowship. I thank Quan-Xing Liu and Johan van de Koppel for helpful comments on the manuscript.

\section{Appendix A. Details of stability changes}

In this Appendix I give some details of the changes in pattern stability that occur in the Klausmeier model (1) and the van de Koppel model (5). These details have no significance for the hysteresis results that are the focus of this paper, and I include them for mathematical completeness.

Periodic travelling wave solutions of partial differential equations can change stability in two different ways (Rademacher and Scheel, 2007). An Eckhaus (sideband) stability change occurs when the curvature of the essential spectrum at the origin changes sign. Alternatively, a fold in the spectrum can cross the imaginary axis at a point away from the origin; this is known as a stability change of Hopf type. For the Klausmeier model (1), all points on the boundary of the pattern stability region are of Eckhaus type (see Figure 3 of Sherratt (2012) for typical plots of the essential spectrum). However for the van de Koppel model (5) the lower part of the stability boundary is of Eckhaus type, while the upper part is of Hopf type. This is indicated in Fig. 4 by dashed lines with long and short dashes respectively. Fig. A.1 shows the essential spectrum of the pattern with $\delta=0.155$, and with $\alpha, \beta$ and $\gamma$ as in Fig. 4, for 4 different values of the migration speed $c$. The pattern is unstable for the values of $c$ used in (a) and (d), and it is stable for (b) and (c). The stability change between (a) and (b) is of Hopf type, while that between (c) and (d) is of Eckhaus type.

\section{Appendix B. Error estimation for numerical simulations}

This paper depends heavily on the results of numerical simulations of (1) and (5), and therefore a detailed estimation of the errors in the numerical scheme is essential. For both systems of equations I used a simple finite difference scheme, in which the diffusion terms are evaluated semi-implicitly, with explicit evaluation of the reaction and advection terms, using upwinding for the latter. I used a uniform grid spacing $\Delta x$ and time step $\Delta t$. As is typical for such a scheme, numerical tests showed that convergence requires that the Courant number $\mathcal{C}$ satisfies the CFL condition $\mathcal{C}<1$; here $\mathcal{C}=v \Delta t / \Delta x$ for the Klausmeier model (1) and $\mathcal{C}=\beta \Delta t / \Delta x$ for the van de Koppel model (5). To investigate numerical accuracy when this condition is satisfied, I used test problems. I fixed the domain length at 50 for (1) and 100 for (5), with periodic boundary conditions. I solved for $0 \leq t \leq 300$, with initial conditions consisting of a small perturbation (the same for each run) to the homogeneous steady state (3) for (1) and (6) for (5). The parameter values were $A=2.5, B=0.45, v=182.5$ for (1) and $\alpha=0.6667, \beta=41.5692, \gamma=0.1333, \delta=0.15$ for (5). As a measure of error, I used the spatial average of the $L_{2}$ norm of the relative error of the solution, compared to a reference solution calculated with high accuracy. Typically the difference in numerical discretisation between the solution being considered and the reference solution results in a spatial translation of the pattern. It is not appropriate to regard this "phase shift" as numerical error, and therefore I performed an appropriate spatial translation before comparing the solutions.

Tables B. 1 and B. 2 show the way in which the error varies with the space and time steps. As is typical for reaction-diffusionconvection problems, the error decreases linearly with $\Delta x$ and $\Delta t$ when these are varied in a constant ratio, so that the Courant number is constant $(<1)$. Moreover, for given $\Delta x$ the error is essentially independent of $\Delta t$ (compare the columns within Tables B.1 and B.2). To investigate this further, I studied numerical convergence in the Klausmeier model (1) as $\Delta x$ and the parameter $v$ were varied, with $\Delta t$ chosen to maintain a CFL number of 0.8 . I also changed $A$ in proportion to $\sqrt{v}$, which maintains the qualitative form of the solution (see Sherratt, 2013b); $B$ and the other solution details were constant, as discussed above. The results in Table B. 3 show that the error is proportional to $\Delta x$ but is essentially independent of $v$.

For the long-term simulations discussed in the main text, the choice of numerical parameters is of course a trade-off between accuracy and CPU time. All of the simulations shown in the main part of this paper used a CFL number of 0.8 , with $\Delta x=0.025$ for ( 1 ) and $\Delta x=0.08$ for (5). In my test problems, this gives errors of about $0.06 \%$ for (1) $0.055 \%$ for (5). 
(a) $c=0.485$

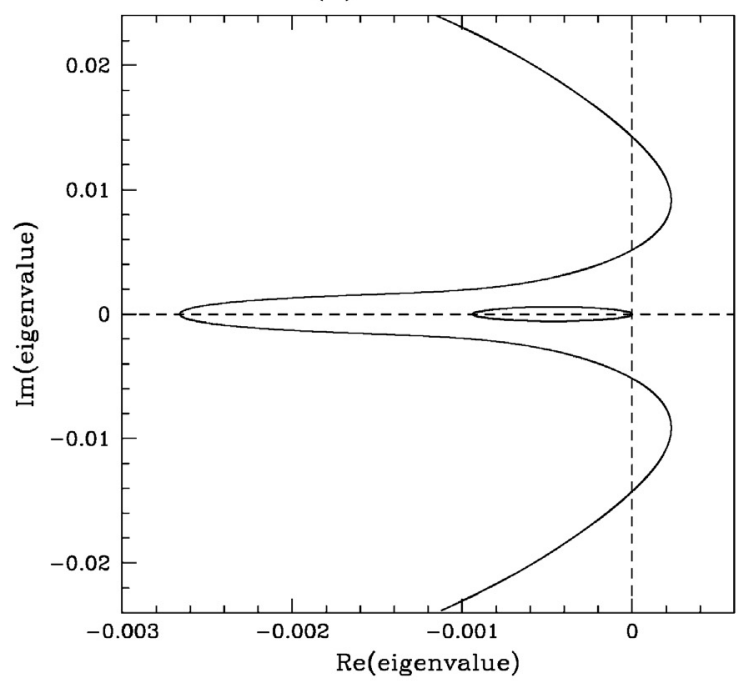

(c) $c=0.15$

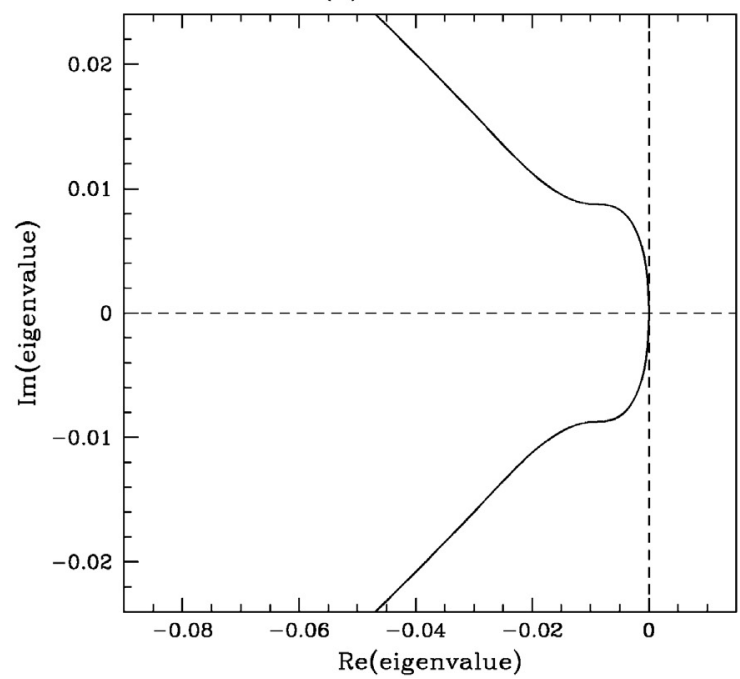

(b) $\quad c=0.48$

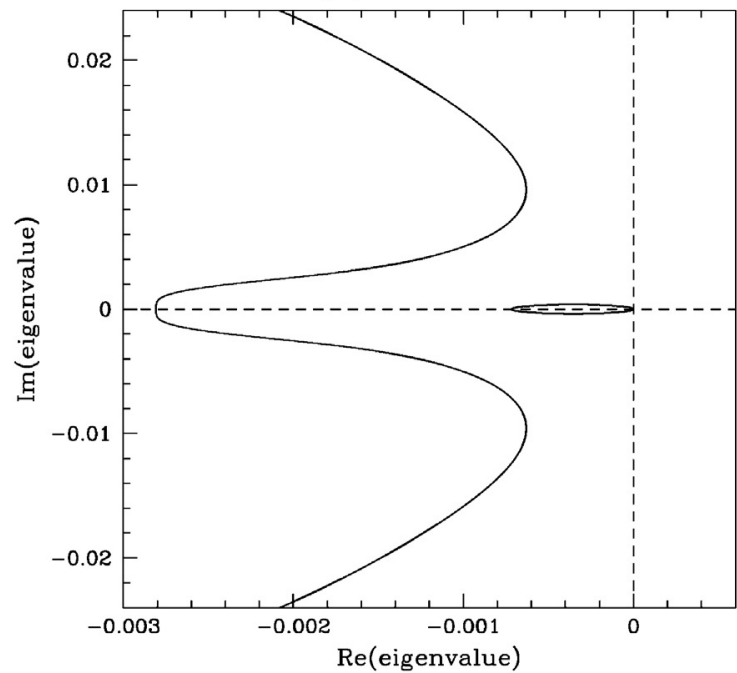

(d) $c=0.08$

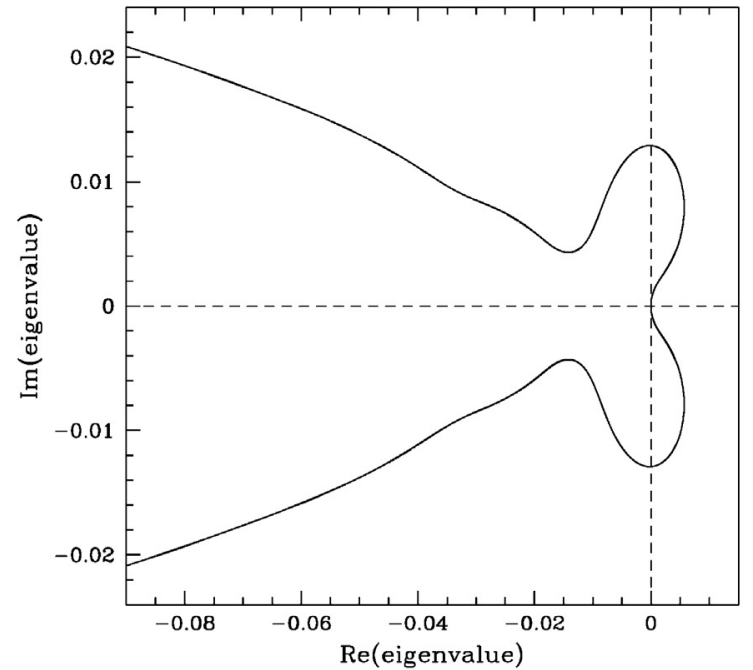

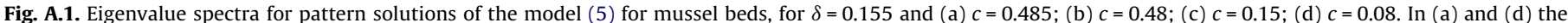

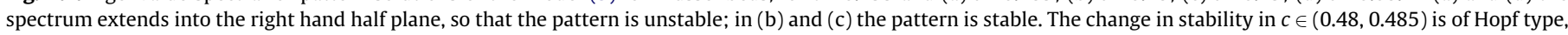

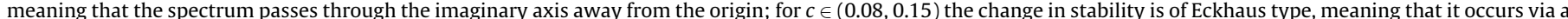

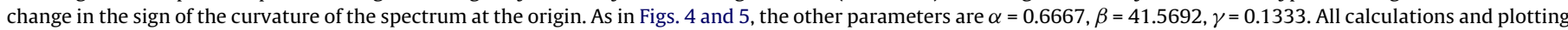

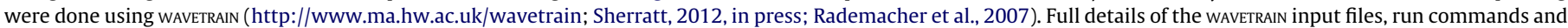
plot commands are given at http://www.ma.hw.ac.uk/ jas/supplements/hysteresis/.

\section{Table B.1}

Convergence of my numerical scheme for the model (1) for pattern formation in vegetation in semi-arid environments. I tabulate the spatial average of the $L_{2}$ norm of the relative error of the solution, compared to the most accurate solution, as a function of the grid spacing $\Delta x$ and the CFL number. Details of the test problem are given in the text.

\begin{tabular}{lllll}
\hline & $\mathrm{CFL}=0.90$ & $\mathrm{CFL}=0.75$ & $\mathrm{CFL}=0.50$ & $\mathrm{CFL}=0.25$ \\
\hline$\Delta x=0.20000$ & 0.00561208 & 0.00561397 & 0.00561704 & 0.00562029 \\
$\Delta x=0.10000$ & 0.00280671 & 0.00280784 & 0.00281000 & 0.00281220 \\
$\Delta x=0.05000$ & 0.00133085 & 0.00133163 & 0.00133286 & 0.00133407 \\
$\Delta x=0.02500$ & 0.00057433 & 0.00057479 & 0.00057540 & 0.00057600 \\
$\Delta x=0.01250$ & 0.00019202 & 0.00019218 & 0.00019246 & 0.00019276 \\
$\Delta x=0.00625$ & 0.00000129 & 0.00000099 & 0.00000050 & 0.00000000 \\
\hline
\end{tabular}

Table B.2

Convergence of my numerical scheme for the model (5) for pattern formation in mussel beds. I tabulate the spatial average of the $L_{2}$ norm of the relative error of the solution, compared to the most accurate solution, as a function of the grid spacing $\Delta x$ and the CFL number. Details of the test problem are given in the text.

\begin{tabular}{llll}
\hline & CFL $=0.90$ & CFL $=0.60$ & CFL $=0.30$ \\
\hline$\Delta x=0.40000$ & 0.00287475 & 0.00285581 & 0.00284171 \\
$\Delta x=0.20000$ & 0.00142499 & 0.00141306 & 0.00140840 \\
$\Delta x=0.10000$ & 0.00069432 & 0.00068832 & 0.00068598 \\
$\Delta x=0.05000$ & 0.00032489 & 0.00032188 & 0.00032071 \\
$\Delta x=0.02500$ & 0.00013977 & 0.00013826 & 0.00013767 \\
$\Delta x=0.01250$ & 0.00004696 & 0.00004621 & 0.00004591 \\
$\Delta x=0.00625$ & 0.00000053 & 0.00000015 & 0.00000000 \\
\hline
\end{tabular}


Table B.3

Convergence of my numerical scheme for the model (1) for pattern formation in vegetation in semi-arid environments. I tabulate the spatial average of the $L_{2}$ norm of the relative error of the solution, compared to the most accurate solution, as a function of the grid spacing $\Delta x$ and $v$, with $A$ varied in proportion to $\sqrt{v}$, which maintains the qualitative form of the solution (see Sherratt, 2013b). Details of the test problem are given in the text.

\begin{tabular}{llll}
\hline & $\nu=100.000$ & $\nu=200.000$ & $v=400.000$ \\
& $A=1.768$ & $A=2.500$ & $A=3.535$ \\
\hline$\Delta x=0.20000$ & 0.00565860 & 0.00576688 & 0.00580979 \\
$\Delta x=0.10000$ & 0.00282260 & 0.00287261 & 0.00289530 \\
$\Delta x=0.05000$ & 0.00133843 & 0.00136279 & 0.00137368 \\
$\Delta x=0.02500$ & 0.00057829 & 0.00058899 & 0.00059313 \\
$\Delta x=0.01250$ & 0.00019344 & 0.00019689 & 0.00019847 \\
$\Delta x=0.00625$ & 0.00000000 & 0.00000000 & 0.00000000 \\
\hline
\end{tabular}

\section{References}

Barbier, N., Couteron, P., Lejoly, J., Deblauwe, V., Lejeune, O., 2006. Self-organized vegetation patterning as a fingerprint of climate and human impact on semi-arid ecosystems. Journal of Ecology 94, 537-547.

Bekker, M.F., Clark, J.T., Jackson, M.W., 2009. Landscape metrics indicate differences in patterns and dominant controls of ribbon forests in the Rocky Mountains, USA. Applied Vegetation Science 12, 237-249.

Belyea, L.R., 2007. Climatic and topographic limits to the abundance of bog pools Hydrological Processes 21, 675-687.

Ben Wu, X., Archer, S.R., 2005. Scale-dependent influence of topography-based hydrologic features on patterns of woody plant encroachment in savanna landscapes. Landscape Ecology 20, 733-742.

Berg, S.S., Dunkerley, D.L., 2004. Patterned mulga near Alice Springs, central Australia, and the potential threat of firewood collection on this vegetation community. Journal of Arid Environments 59, 313-350.

Borgogno, F., D'Odorico, P., Laio, F., Ridolfi, L., 2009. Mathematical models of vegetation pattern formation in ecohydrology. Reviews of Geophysics 47, RG1005.

Couteron, P., Mahamane, A., Ouedraogo, P., Seghieri, J., 2000. Differences between banded thickets (tiger bush) at two sites in West Africa. Journal of Vegetation Science 11, 321-328.

Deblauwe, V., 2010. Modulation des structures de végétation auto-organisées en milieu aride Self-organized vegetation pattern modulation in arid climates. $\mathrm{PhD}$ Thesis. Université Libre de Bruxelles. http://theses.ulb.ac.be/ETD-db/collection/ available/ULBetd-04122010-093151/.

Deblauwe, V., Couteron, P., Lejeune, O., Bogaert, J., Barbier, N., 2011. Environmental modulation of self-organized periodic vegetation patterns in Sudan. Ecography 34, 990-1001.

Deblauwe, V., Barbier, N., Couteron, P., Lejeune, O., Bogaert, J., 2008. The global biogeography of semi-arid periodic vegetation patterns. Global Ecology and Biogeography 17, 715-723.

Denny, M.W., 1987. Lift as a mechanism of patch initiation in mussel beds. Journal of Experimental Marine Biology And Ecology 113, 231-246.

Dunkerley, D.L., Brown, K.J., 2002. Oblique vegetation banding in the Australian arid zone: implications for theories of pattern evolution and maintenance. Journal of Arid Environments 52, 163-181.

Eppinga, M.B., de Ruiter, P.C., Wassen, M.J., Rietkerk, M., 2009. Nutrients and hydrology indicate the driving mechanisms of peatland surface patterning. American Naturalist 173, 803-818.

Gilad, E., von Hardenberg, J., Provenzale, A., Shachak, M., Meron, E., 2007. A mathematical model of plants as ecosystem engineers. Journal of Theoretical Biology 244, 680-691.

Guichard, F., Halpin, P.M., Allison, G.W., Lubchenco, J., Menge, B.A., 2003. Mussel disturbance dynamics: signatures of oceanographic forcing from local interactions. American Naturalist 161, 889-904.

Hemming, C.F., 1965. Vegetation arcs in Somaliland. Journal of Ecology 53 57-67.

Hiemstra, C.A., Liston, G.E., Reiners, W.A., 2006. Observing, modelling, and validating snow redistribution by wind in a Wyoming upper treeline landscape. Ecological Modelling 197, 35-51

HilleRisLambers, R., Rietkerk, M., van de Bosch, F., Prins, H.H.T., de Kroon, H., 2001. Vegetation pattern formation in semi-arid grazing systems. Ecology 82, 50-61.

Klausmeier, C.A., 1999. Regular and irregular patterns in semiarid vegetation. Science 284, 1826-1828.

Kopell, N., Howard, L.N., 1973. Plane wave solutions to reaction-diffusion equations. Studies in Applied Mathematics 52, 291-328.

Lefever, R., Lejeune, O., 1997. On the origin of tiger bush. Bulletin of Mathematical Biology 59, 263-294.

Lejeune, O., Tlidi, M., Couteron, P., 2002. Localized vegetation patches: a selforganized response to resource scarcity. Physical Review E 66, 010901.

Leprun, J.C., 1999. The influences of ecological factors on tiger bush and dotted bush patterns along a gradient from Mali to northern Burkina Faso. Catena 37 25-44.
Lesschen, J.P., Cammeraat, L.H., Kooijman, A.M., van Wesemael, B., 2008. Development of spatial heterogeneity in vegetation and soil properties after land abandonment in a semi-arid ecosystem. Journal of Arid Environments 72, 2082-2092.

Liu, Q.-X., Weerman, E.J., Herman, P.M.J., Olff, H., van de Koppel, J., 2012. Alternative mechanisms alter the emergent properties of self-organization in mussel beds. Proceedings of the Royal Society of London Series B 279, 2744-2753.

Mabbutt, J.A., Fanning, P.C., 1987. Vegetation banding in arid Western Australia. Journal of Arid Environments 12, 41-59.

McDonald, A.K., Kinucan, R.J., Loomis, L.E., 2009. Ecohydrological interactions within banded vegetation in the northeastern Chihuahuan Desert, USA. Ecohydrology 2, 66-71.

MacFadyen, W., 1950. Vegetation patterns in the semi-desert plains of British Somaliland. Geographical Journal 115, 199-211.

Montaña, C., 1992. The colonization of bare areas in two-phase mosaics of an arid ecosystem. Journal of Ecology 80, 315-327.

Montaña, C., Seghieri, J., Cornet, A., 2001. Vegetation dynamics: recruitment and regeneration in two-phase mosaics. In: Tongway, D.J., Valentin, C., Seghieri, J. (Eds.), Banded Vegetation Patterning in Arid and Semi-Arid Environments. Springer, New York, pp. 132-145.

Paine, R.T., Levin, S.A., 1981. Intertidal landscapes: disturbance and the dynamics of pattern. Ecological Monographs 51, 145-178.

Rademacher, J.D.M., Scheel, A., 2007. Instabilities of wave trains and turing patterns in large domains. International Journal of Bifurcation and Chaos 17, 2679-2691.

Rademacher, J.D.M., Sandstede, B., Scheel, A., 2007. Computing absolute and essential spectra using continuation. Physica D 229, 166-183.

Rietkerk, M., van de Koppel, J., 2008. Regular pattern formation in real ecosystems. TREE 23, 169-175.

Rietkerk, M., Dekker, S.C., de Ruiter, P.C., van de Koppel, J., 2004. Self-organized patchiness and catastrophic shifts in ecosystems. Science 305, 19261929.

Rietkerk, M., Boerlijst, M.C., van Langevelde, F., HilleRisLambers, R., van de Koppel, J., Prins, H.H.T., de Roos, A., 2002. Self-organisation of vegetation in arid ecosystems. American Naturalist 160, 524-530.

Robles, C.D., Garza, C., Desharnais, R.A., Donahuey, M.J., 2010. Landscape patterns in boundary intensity: a case study of mussel beds. Landscape Ecology 25, 745759.

Saco, P.M., Willgoose, G.R., Hancock, G.R., 2007. Eco-geomorphology of banded vegetation patterns in arid and semi-arid regions. Hydrology and Earth System Sciences $11,1717-1730$.

Sherratt, J.A., 2005. An analysis of vegetation stripe formation in semi-arid landscapes. Journal of Mathematical Biology 51, 183-197.

Sherratt, J.A., 2010. Pattern solutions of the Klausmeier model for banded vegetation in semi-arid environments I. Nonlinearity 23, 2657-2675.

Sherratt, J.A., 2011. Pattern solutions of the Klausmeier model for banded vegetation in semi-arid environments II. Patterns with the largest possible propagation speeds. Proceedings of the Royal Society of London Series A 467, 32723294.

Sherratt, J.A., 2012. Numerical continuation methods for studying periodic travelling wave (wavetrain) solutions of partial differential equations. Applied Mathematics and Computation 218, 4684-4694.

Sherratt, J.A., 2013a. Pattern solutions of the Klausmeier model for banded vegetation semi-arid environments III: the transition between homoclinic solutions. Physica D 242, 30-41.

Sherratt, J.A., 2013b. Pattern solutions of the Klausmeier model for banded vegetation semi-arid environments IV: slowly moving patterns and their stability. SIAM Journal on Applied Mathematics 73, 330-350.

Sherratt, J.A. Numerical continuation of boundaries in parameter space between stable and unstable periodic travelling wave (wavetrain) solutions of partial differential equations. Advances in Computational Mathematics, http:// dx.doi.org/10.1007/s10444-012-9273-0, in press.

Sherratt, J.A., Lord, G.J., 2007. Nonlinear dynamics and pattern bifurcations in a model for vegetation stripes in semi-arid environments. Theoretical Population Biology 71, 1-11.

Sherratt, J.A., Smith, M.J., 2008. Periodic travelling waves in cyclic populations: field studies and reaction-diffusion models. Journal of the Royal Society, Interface 5, 483-505.

Snover, M.L., Commito, J.A., 1998. The fracal geometry of Mytilus edulis L. spatial distribution in a soft-bottom system. Journal of Experimental Marine Biology and Ecology 223, 53-64.

Thompson, S., Katul, G., 2009. Secondary seed dispersal and its role in landscape organization. Geophysical Research Letters 36, L02402.

Tongway, D.J., Ludwig, J.A., 2001. Theories on the origins, maintainance, dynamics, and functioning of banded landscapes. In: Tongway, D.J., Valentin, C., Seghieri, J. (Eds.), Banded Vegetation Patterning in Arid and Semi-Arid Environments. Springer, New York, pp. 20-31.

Ursino, N., 2005. The influence of soil properties on the formation of unstable vegetation patterns on hillsides of semiarid catchments. Advances in Water Resources 28, 956-963.

Valentin, C., d'Herbès, J.M., 1999. Niger tiger bush as a natural water harvesting system. Catena 37, 231-256.

Valentin, C., d'Herbès, J.M., Poesen, J., 1999. Soil and water components of banded vegetation patterns. Catena 37, 1-24.

van de Koppel, J., Rietkerk, M., Dankers, N., Herman, P.M.J., 2005. Scale-dependent feedback and regular spatial patterns in young mussel beds. American Naturalist 165, E66-E77. 
van de Koppel, J., Crain, C.M., 2006. Scale-dependent inhibition drives regular tussock spacing in a freshwater marsh. American Naturalist 168, E136-E147.

van de Koppel, J., Gascoigne, J.C., Theraulaz, G., Rietkerk, M., Mooij, W.M., Herman, P.M.J., 2008. Experimental evidence for spatial self-organisation and its emergent effects in mussel bed ecosystems. Science 322, 739-742.

von Hardenberg, J., Kletter, A.Y., Yizhaq, H., Nathan, J., Meron, E., 2010. Periodic versus scale-free patterns in dryland vegetation. Proceedings of the Royal Society of London Series B 277, 1771-1776.
Wang, R.H., Liu, Q.X., Sun, G.Q., Jin, Z., van de Koppel, J., 2009. Nonlinear dynamic and pattern bifurcations in a model for spatial patterns in young mussel beds. Journal of the Royal Society, Interface 6, 705-718.

Wootton, J.T., 2001. Local interactions predict large-scale pattern in empirically derived cellular automata. Nature 413, 841-844.

Worrall, G.A., 1959. The Butana grass patterns. Journal of Soil Science 10, 34-53.

Yu, B.G., 2010. Dynamic behaviour of a plant-wrack model with spatial diffusion. Communications in Nonlinear Science and Numerical Simulation 15, 2201 2205. 